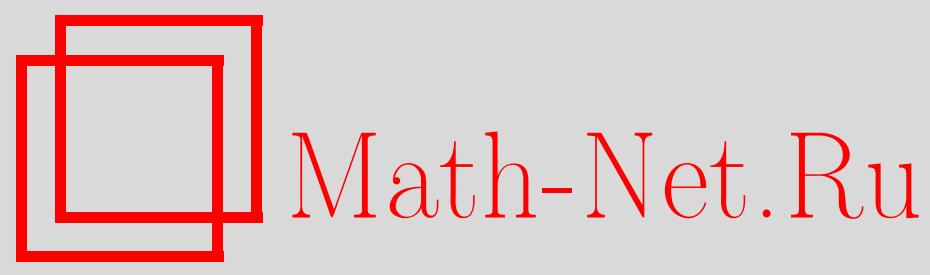

В. Ю. Попов, О разрешимости эквациональных теорий многообразий колец, Матем. заметки, 1998, том 63, выпуск $6,873-881$

DOI: https://doi.org/10.4213/mzm1358

Использование Общероссийского математического портала Math-Net.Ru подразумевает, что вы прочитали и согласны с пользовательским соглашением http://www.mathnet.ru/rus/agreement

Параметры загрузки:

IP: 54.92 .164 .108

26 апреля 2023 г., 16:51:03

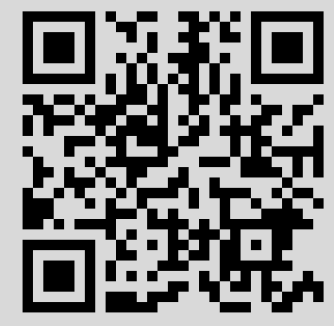




\section{О РАЗРЕШИМОСТИ ЭКВАЦИОНАЛЬНЫХ ТЕОРИЙ МНОГООБРАЗИЙ КОЛЕЦ}

\section{В. Ю. Попов}

Получен результат, иллюстрирующий сложность описания многообразий колец с неразрешимой эквациональной теорией.

Библиография: 7 названий.

В работе [1] А. И. Мальцев построил конечно базируемое многообразие квазигрупा с неразрешимой эквациональной теорией и поставил опубликованньй в [2] вопрос о существовании конечно базируемых многообразий полугрупп, групп и колец с неразрешимой эквациональной теорией. Положительньй ответ на этот вопрос для полугруш получен в [3], а для групп - в [4]. В работе [5] построены примеры конечно базируемых многообразий неассоциативных колец с неразрешимой эквациональной теорией. Следующий результат иллюстрирует сложность описания многообразий колец с неразрешимой эквациональной теорией.

ТЕОремА. Существует последовательность конечно базируемых многообразий неассоииативных колеи, $\mathfrak{A}_{1} \subset \mathfrak{B}_{1} \subset \mathfrak{A}_{2} \subset \mathfrak{B}_{2} \subset \cdots$ такая, что для всех $i$ әквациональная теория $\mathfrak{A}_{i}$ неразрешима, а әквациональная теория $\mathfrak{B}_{i}$ разрешима.

Из теоремы вытекает утверждение, решающее поставленную в общем случае в работе [6] проблему описания SA-критических теорий для многообразий $\mathfrak{A}_{i}$.

СлЕДСТВИЕ. Теория $\forall \mathfrak{A}_{i}$ является единственной критической теорией многообразия $\mathfrak{A}_{i}$.

ДоКАЗАТЕЛЬСТВО ТЕОРЕМЫ. Введем ряд обозначений для слов свободного группоида Г со свободным порождаюшим множеством $e, e_{1}, e_{2}, \ldots, e_{n}, \ldots$ Пусть

$$
\begin{gathered}
s\left(x_{1}, \ldots, x_{k}\right)=\left(\left(x_{k-1}\left(\left(\ldots\left(x_{4}\left(\left(x_{1} x_{2}\right) x_{3}\right)\right) x_{5} \ldots\right) x_{k-2}\right)\right) x_{k}\right), \\
t\left(x_{1}, \ldots, x_{k+l}\right)=s\left(s\left(x_{1}, \ldots, x_{k}\right), x_{k+1}, \ldots, x_{k+l}\right) .
\end{gathered}
$$

Если $w\left(e_{1}, \ldots, e_{k}\right) \in \Gamma$, то через $w_{k}(x)$ обозначим слово, полученное из $w\left(e_{1}, \ldots, e_{k}\right)$ подстановкой

$$
\left(\begin{array}{ccc}
e_{1} & \ldots & e_{k} \\
x & \ldots & x
\end{array}\right) .
$$

Пусть

$$
\begin{gathered}
A(x)=t_{k+17}(x), \quad B(x)=t_{k+18}(x), \quad C(x)=t_{k+19}(x), \quad D(x)=t_{k+20}(x), \\
\\
Q_{i}(x)=t_{k+i+21}(x),
\end{gathered}
$$


где $i \in \omega, k$ - фиксированное натуральное число такое, что $k \geqslant 40$. Будем считать, если не оговорено противное, что

$$
w_{1} \ldots w_{n}=\left(\ldots\left(\left(w_{1} w_{2}\right) w_{3}\right) \ldots\right) w_{n}, \quad w_{1} w_{2}^{n}=\left(\ldots\left(\left(w_{1} w_{2}\right) w_{2}\right) \ldots\right) w_{2}
$$

и $y^{0}-$ пустой символ.

Пусть $P$ - некоторое рекурсивно перечислимое нерекурсивное множество натуральных чисел. Обозначим через М двухленточную машину Минского, вычисляющую частичную характеристическую функцию множества $P$. Произвольной команде

$$
q_{i} \delta_{1} \delta_{2} \rightarrow q_{j} \varepsilon_{1} \varepsilon_{2}
$$

машины М сопоставим кодируюшее ее тождество:

$$
\begin{array}{ll}
Q_{i}(x) C(x) D(x)=Q_{j}(x) A^{\varepsilon_{1}}(x) B^{\varepsilon_{2}}(x) C(x) D(x), & \text { если } \delta_{1}=1, \delta_{2}=1, \\
Q_{i}(x) A(x) D(x)=Q_{j}(x) A^{\varepsilon_{1}+1}(x) B^{\varepsilon_{2}}(x) D(x), & \text { если } \delta_{1}=1, \delta_{2}=0, \\
Q_{i}(x) B(x) C(x)=Q_{j}(x) A^{\varepsilon_{1}}(x) B^{\varepsilon_{2}+1}(x) C(x), & \text { если } \delta_{1}=0, \delta_{2}=1, \\
Q_{i}(x) A(x) B(x)=Q_{j}(x) A^{\varepsilon_{1}+1}(x) B^{\varepsilon_{2}+1}(x), & \text { если } \delta_{1}=0, \delta_{2}=0 .
\end{array}
$$

Пусть число внутренних состояний машины $\mathrm{M}$ равно $m+1$,

$$
W=\left\{A(x), B(x), C(x), D(x), Q_{i}(x) \mid 0 \leqslant i \leqslant m\right\} .
$$

Обозначим через $\mathfrak{X}_{k}^{1}$ многообразие колец, заданное тождествами, кодирующими команды машины M, а через $\mathfrak{X}_{k}^{2}$ многообразие колец, заданное тождествами

$$
\begin{gathered}
y F(x) B(x)=y B(x) A(x), \quad y C(x) D(x)=y D(x) C(x), \quad y A(x) D(x)=y D(x) A(x), \\
y B(x) C(x)=y C(x) B(x), \quad z\left(\left(\ldots\left(\left(y x_{1}\right) x_{2}\right) \ldots\right) x_{r}\right)=0,
\end{gathered}
$$

где $r=v+k+100$.

Пусть $\mathfrak{R}_{2}$ - многообразие всех колец характеристики 2 и $\mathfrak{X}_{k}=\mathfrak{X}_{k}^{1} \cap \mathfrak{X}_{k}^{2} \cap \mathfrak{R}_{2}$. Обозначим через $F \mathfrak{R}_{2}, F \mathfrak{X}_{k}, F \mathfrak{X}_{k}^{i}$ кольца счетного ранга, свободные в многообразиях $\mathfrak{R}_{2}$, $\mathfrak{X}_{k}, \mathfrak{X}_{k}^{i}$ соответственно, а через $\left\{e, e_{1}, e_{2}, \ldots, e_{n}, \ldots\right\}$ множество свободных образующих кольца $F \mathfrak{R}_{2}$. Для удобства свободные образующие колец $F \mathfrak{X}_{k}$ и $F \mathfrak{X}_{k}^{i}$ будем обозначать теми же буквами.

Далее нам потребуются три леммы.

Лемма 1. Пусть $w_{1}(e) \ldots w_{n}(e)$ - слово из свободного группоида $\Gamma, w_{i}(x) \in W$. Тогда слово $w_{1}(e) \ldots w_{n}(e)$ содержит подслово $t\left(y_{1}, \ldots, y_{k+l}\right)$ для $y_{i}=e^{p_{i}} u l>16$, если и только если $i$ такое, что $w_{i}(e)=t\left(y_{1}, \ldots, y_{k+l}\right)$. 
ДокАЗАТЕЛЬСтво. Очевидно, что если существует $i$ такое, что $w_{i}(e)=t\left(y_{1}, \ldots\right.$, $\left.y_{k+l}\right)$, то $t\left(y_{1}, \ldots, y_{k+l}\right)$ - подслово слова $w_{1}(e) \ldots w_{n}(e)$. Докажем индукцией по $n$, что $w_{i}(e)=t\left(y_{1}, \ldots, y_{k+l}\right)$ для некоторого $i$. Пусть $n=1$. Тогда $t\left(y_{1}, \ldots, y_{k+l}\right)-$ подслово слова $w_{1}(e)$. Очевидно, что $t\left(y_{1}, \ldots, y_{k+l}\right)$ в силу своего строения не может быть собственным подсловом слова $w_{1}(e)$. Следовательно, $w_{1}(e)=t\left(y_{1}, \ldots, y_{k+l}\right)$. Предположим, что утверждение справедливо для некоторого $n$. Покажем, что оно верно и для $n+1$. В самом деле, пусть $t\left(y_{1}, \ldots, y_{k+l}\right)$ - подслово слова $w_{1}(e) \ldots w_{n+1}(e)$. Заметим, что по определению всякое собственноеподслово слова $w_{1}(e) \ldots w_{n+1}(e)$ есть либо подслово слова $w_{1}(e) \ldots w_{n}(e)$, либо подслово слова $w_{n+1}(e)$. Поэтому по предположению индукции получаем, что $t\left(y_{1}, \ldots, y_{k+l}\right)$ является собственным подсловом слова $w_{1}(e) \ldots w_{n+1}(e)$ тогда и только тогда, когда существует $i$ такое, что $t\left(y_{1}, \ldots, y_{k+l}\right)=$ $w_{i}(e)$. Пусть теперь $t\left(y_{1}, \ldots, y_{k+l}\right)=w_{1}(e) \ldots w_{n+1}(e)$. Заметим, что $t\left(y_{1}, \ldots, y_{k+l}\right)=$ $\left(\left(y_{k+l-1} t^{*}\left(y_{1}, \ldots, y_{k+l-2}\right)\right) y_{k+l}\right)$. Следовательно, слово $w_{1}(e) \ldots w_{n+1}(e)$ представимо в виде $\left(z_{1} z_{2}\right) z_{3}$, где $z_{i}$ - некоторое слово от $e$. Легко убедиться, что $\left(z_{1} z_{2}\right) z_{3}=w_{1}(e) \ldots$ $w_{n+1}(e)$ тогда и только тогда, когда

$$
z_{1}=w_{1}(e) \ldots w_{n-1}(e), \quad z_{2}=w_{n}(e), \quad z_{3}=w_{n+1}(e)
$$

соответственно. Поэтому $t^{*}\left(y_{1}, \ldots, y_{k+l-2}\right)=w_{n}(e)$.

Так как $l>16$, слово $t^{*}\left(y_{1}, \ldots, y_{k+l-2}\right)$ представимо в виде

$$
\left(\left(\left(t^{* *}\left(y_{1}, \ldots, y_{k+l-5}\right) y_{k+l-4}\right) y_{k+l-3}\right) y_{k+l-2}\right)
$$

Следовательно, слово $w_{n}(e)$ должно быть представимо в виде $\left(\left(\left(u_{1} u_{2}\right) u_{3}\right) u_{4}\right)$. Но, как нетрудно убедиться, слово $w_{n}(e)$ в таком виде представить нельзя. Полученное противоречие доказьвает, что $t\left(y_{1}, \ldots, y_{k+l}\right)$ - подслово слова $w_{1}(e) \ldots w_{n+1}(e)$ тогда и только тогда, когда $t\left(y_{1}, \ldots, y_{k+l}\right)=w_{i}(e)$ для некоторого $i$.

Лемма 1 доказана.

Лемма 2. Если существует конечное вычисление на машине $\mathrm{M}$, переводящее конфигурацию $q_{i} \xi_{1} \xi_{2}$ в конфигурацию $q_{j} \eta_{1} \eta_{2}$, то в многообразии $\mathfrak{X}_{k}$ выполняется тохсдество

$$
Q_{i}(x) A^{\xi_{1}}(x) B^{\xi_{2}}(x) C(x) D(x)=Q_{j}(x) A^{\eta_{1}}(x) B^{\eta_{2}}(x) C(x) D(x) .
$$

ДокАЗАТЕЛЬСТво. Пусть машина М через $d$ шагов переходит из конфигурации $q_{i} \xi_{1} \xi_{2}$ в конфигурацию $q_{j} \eta_{1} \eta_{2}$. Индукцией по числу $d$ докажем, что в многообразии $\mathfrak{X}_{k}$ выполняется тождество (1). Пусть $d=0$. Тогда $i=j, \xi_{1}=\eta_{1}, \xi_{2}=\eta_{2}$. Следовательно, тождество (1) тривиальное. База индукции проверена.

Допустим, что утверждение справедливо для некоторого $d$. Покажем, что оно верно и для $d+1$. Пусть через $d$ тактов работы машина М перевела конфигурацию $q_{i} \xi_{1} \xi_{2}$ в конфигурацию $q_{j} \eta_{1} \eta_{2}$ и на $(d+1)$-м шаге выполняется команда

$$
q_{j} \delta_{1} \delta_{2} \rightarrow q_{l} \alpha \beta
$$

В зависимости от значений $\delta_{1}$ и $\delta_{2}$ возможны четыре случая. Рассмотрим лишь случай, когда $\delta_{1}=1$ и $\delta_{2}=0$, так как остальные три рассматриваются аналогично. Поскольку 
на $(d+1)$-м шаге выполняется команда $q_{j} 10 \rightarrow q_{l} \alpha \beta$, то $\eta_{1}=0, \eta_{2} \neq 0$ и в многообразии $\mathfrak{X}_{k}$ вьполняется тождество

$$
Q_{j}(x) B(x) C(x)=Q_{l}(x) A^{\alpha}(x) B^{1+\beta}(x) C(x) .
$$

Таким образом, получаем, что

$$
\begin{aligned}
& Q_{i}(x) A^{\xi_{1}}(x) B^{\xi_{2}}(x) C(x) D(x)=Q_{j}(x) A^{\eta_{1}}(x) B^{\eta_{2}}(x) C(x) D(x) \\
& \quad=Q_{j}(x) A^{\eta_{1}}(x) B(x) C(x) B^{\eta_{2}-1}(x) D(x)=Q_{j}(x) B(x) C(x) B^{\eta_{2}-1}(x) D(x) \\
& \quad=Q_{l}(x) A^{\alpha}(x) B^{1+\beta}(x) C(x) B^{\eta_{2}-1}(x) D(x)=Q_{l}(x) A^{\alpha}(x) B^{\eta_{2}+\beta}(x) C(x) D(x) \\
& \quad=Q_{l}(x) A^{\eta_{1}+\alpha}(x) B^{\eta_{2}+\beta}(x) C(x) D(x) .
\end{aligned}
$$

При этом первое равенство справедливо по предположению индукции, второе и пятое выполняются в силу тождества $y B(x) C(x)=y C(x) B(x)$, третье и шестое имеют место, так как $\eta_{1}=0$, а четвертое - в силу тождества (2).

Лемма 2 доказана.

Лемма 3. Пусть в многообразии $\mathfrak{X}_{k}$ выполняется тождество (1). Тогда существует конечное вычисление на $\mathrm{M}$, переводящее конфигурацию $q_{j} \eta_{1} \eta_{2}$ в конфигурацию $q_{i} \xi_{1} \xi_{2}$, или наоборот.

ДокАЗАТЕЛЬСТво. Покажем сначала, что если $w_{1}(e) \ldots w_{n}(e)$ - элемент свободного группоида $\Gamma$, где $w_{i}(x) \in W$, то слово $w_{1}(e) \ldots w_{n}(e)$ не содержит подслов вида

$$
Y\left(x_{1}, \ldots, x_{r}\right)=x_{r}\left(\left(\ldots\left(\left(x_{1} x_{2}\right) x_{3}\right) \ldots\right) x_{r-1}\right)
$$

при $r=m+k+102$. Доказательство этого факта проведем индукцией по $n$. В самом деле, пусть $n=1$. Тогда необходимо показать, что $Y\left(x_{1}, \ldots, x_{r}\right)$ не является подсловом слова $w_{1}(e)$. По построению слова $w_{1}(e)$ его длина не превосходит $k+m+20$. Так как длина слова $Y\left(x_{1}, \ldots, x_{r}\right)$ не меньше $r$, a $r=k+m+102$, слово $Y\left(x_{1}, \ldots, x_{r}\right)$, очевидно, не является подсловом слова $w_{1}(e)$. База индукции проверена.

Допустим, что обсуждаемое утверждение справедливо для некоторого $n$. Покажем, что оно верно и для $n+1$. Очевидно, что всякое собственное подслово слова $w_{1}(e) \ldots$ $w_{n+1}(e)$ является подсловом слова $w_{1}(e) \ldots w_{n}(e)$ или подсловом слова $w_{n+1}(e)$. Следовательно, по предположению индукции $Y\left(x_{1}, \ldots, x_{r}\right)$ не является собственным подсловом слова $w_{1}(e) \ldots w_{n+1}(e)$. Допустим, что $Y\left(x_{1}, \ldots, x_{r}\right)=w_{1}(e) \ldots w_{n+1}(e)$. Тогда, как нетрудно убедиться, должны вьполняться следующие равенства:

$$
x_{r}=w_{1}(e) \ldots w_{n}(e), \quad\left(\ldots\left(\left(x_{1} x_{2}\right) x_{3}\right) \ldots\right) x_{r-1}=w_{n+1}(e) .
$$

Длина слова $\left(\ldots\left(\left(x_{1} x_{2}\right) x_{3}\right) \ldots\right) x_{r-1}$ не меньше, чем $m+k+102$, а длина слова $w_{n+1}(e)$ не превосходит $m+k+20$. Следовательно, равенство

$$
\left(\ldots\left(\left(x_{1} x_{2}\right) x_{3}\right) \ldots\right) x_{r-1}=w_{n+1}(e)
$$

неверно. Полученное противоречие доказывает, что слово $Y\left(x_{1}, \ldots, x_{r}\right)$ не является подсловом слова $w_{1}(e) \ldots w_{n}(e)$. 
Заметим, что

$$
\begin{aligned}
\mathfrak{X}_{k} & =Q_{i}(x) A^{\xi_{1}}(x) B^{\xi_{2}}(x) C(x) D(x)=Q_{j}(x) A^{\eta_{1}}(x) B^{\eta_{2}}(x) C(x) D(x) \\
& \Longleftrightarrow F \mathfrak{X}_{k}=Q_{i}(e) A^{\xi_{1}}(e) B^{\xi_{2}}(e) C(e) D(e)=Q_{j}(e) A^{\eta_{1}}(e) B^{\eta_{2}}(e) C(e) D(e) .
\end{aligned}
$$

Обозначим через $S$ множество многочленов $f(e)$ таких, что $f(e)=0$ - соотношение в кольце $F \mathfrak{X}_{k}$, полученное подстановкой конкретных элементов кольца $F \mathfrak{X}_{k}$ вместо переменных в одно из тождеств, задающих многообразие $\mathfrak{X}_{k}^{1} \cap \mathfrak{X}_{k}^{2}$. Пусть $I$ - идеал в кольце $F \mathfrak{X}_{2}$, порожденньй множеством $S$. Очевидно, что

$$
\begin{aligned}
F \mathfrak{X}_{k} & =Q_{i}(e) A^{\xi_{1}}(e) B^{\xi_{2}}(e) C(e) D(e)=Q_{j}(e) A^{\eta_{1}}(e) B^{\eta_{2}}(e) C(e) D(e) \\
& \Longleftrightarrow Q_{i}(e) A^{\xi_{1}}(e) B^{\xi_{2}}(e) C(e) D(e)+Q_{j}(e) A^{\eta_{1}}(e) B^{\eta_{2}}(e) C(e) D(e) \in I .
\end{aligned}
$$

Вхождение данного многочлена в идеал $I$ равносильно тому, что в кольце $F \Re_{2}$ выполняется равенство вида

$$
Q_{i}(e) A^{\xi_{1}}(e) B^{\xi_{2}}(e) C(e) D(e)+Q_{j}(e) A^{\eta_{1}}(e) B^{\eta_{2}}(e) C(e) D(e)=\sum_{i, j} u_{i 1} h_{j} u_{i 2}
$$

где $h_{j} \in S, u_{i 1} h_{j} u_{i 2}$ - некоторое произведение многочленов, одним из сомножителей в котором является многочлен $h_{j}$. Покажем, что сумма $\sum_{i, j} u_{i 1} h_{j} u_{i 2}$ представима в виде $f_{1}+f_{2}$, где $f_{1}=\sum_{i, j} v_{i 1} h_{j} v_{i 2}$, и, кроме того, каждый одночлен многочлена $f_{1}$ представим в виде $w_{1}(e) \ldots w_{n}(e)$, где $w_{i}(x) \in W$, а $f_{2}-$ многочлен, ни один одночлен которого нельзя представить в виде $w_{1}(e) \ldots w_{n}(e)$. Доказательство проведем индукцией по числу одночленов в сумме $\sum_{i, j} u_{i 1} h_{j} u_{i 2}$. Допустим, что число одночленов в сумме равно 0. Тогда утверждение очевидно. Пусть утверждение справедливо для некоторого числа $d$ одночленов. Покажем, что оно верно и для $d+1$. Пусть $\sum_{i, j} u_{i 1} h_{j} u_{i 1}-$ сумма, число одночленов в которой не превосходит $d+1$. Тогда число одночленов в сумме $\sum_{i>1, j} u_{i 1} h_{j} u_{i 2}$ не превосходит $d$, и в кольце $F \Re_{2}$ выполняется равенство

$$
u_{11} h_{1} u_{12}+\sum_{i>1, j} u_{i 1} h_{j} u_{i 2}=\sum_{i, j} u_{i 1} h_{j} u_{i 2}
$$

Отсюда по предположению индукции получаем, что

$$
\sum_{i, j} u_{i 1} h_{j} u_{i 2}=f_{1}+f_{2}+u_{11} h_{1} u_{12}
$$

Поэтому достаточно показать, что многочлен $u_{11} h_{1} u_{12}$ представим в требуемом виде. Следует рассмотреть два случая в зависимости от того, соответствует ли многочлен $h_{1}$ тождеству многообразия $\mathfrak{X}_{k}^{1}$ или тождеству многообразия $\mathfrak{X}_{k}^{2}$. Допустим, что $h_{1}$ соответствует тождеству $\mathfrak{X}_{k}^{2}$. Тогда он имеет вид $y w_{1}(x) w_{2}(x)+y w_{2}(x) w_{1}(x)$.

В силу дистрибутивности можно считать, что $y$, а также все многочлены, входящие в $u_{11} h_{1} u_{12}$, кроме $h_{1}$, являются одночленами. Допустим, что $x$-многочлен, который не содержит одночлен $e$. Тогда получим, что $w_{1}(x)$ - многочлен, состоящий из одночленов вида $t\left(y_{1}, \ldots, y_{k+l}\right)$, причем ни один из них не принадлежит $W$. Отсюда в силу леммы 1 
получаем, что $f_{1}+\left(f_{2}+u_{11} h_{1} u_{12}\right)$ - требуемое представление суммы $\sum_{i, j} u_{i 1} h_{j} u_{i 2}$. Пусть теперь $x=e+x^{*}$, где $x^{*}$ - многочлен, которьй не содержит одночлен $e$. Тогда

$$
\begin{aligned}
u_{11} h_{1} u_{12} & =u_{11}\left(y w_{1}\left(e+x^{*}\right) w_{2}\left(e+x^{*}\right)+y w_{2}\left(e+x^{*}\right) w_{1}\left(e+x^{*}\right)\right) u_{12} \\
& =u_{11}\left(y w_{1}(e) w_{2}(e)+y w_{2}(e) w_{1}(e)+f\right) u_{12},
\end{aligned}
$$

где $f$ - многочлен, ни один одночлен которого в силу леммы 1 нельзя представить в виде $w_{1}(e) \ldots w_{n}(e)$. Как упомянуто выше, $y$ можно считать одночленом. Если $y$ нельзя представить в виде $w_{1}(e) \ldots w_{n}(e)$, то очевидно, что $f_{1}+\left(f_{2}+u_{11} h_{1} u_{12}\right)$ - требуемое представление. Пусть $e=w_{i_{1}}(e) \ldots w_{i_{k}}(e)$. Тогда

$$
u_{11} h_{1} u_{12}=u_{11}\left(w_{i_{1}}(e) \ldots w_{i_{k}}(e) w_{1}(e) w_{2}(e)+w_{i_{1}}(e) \ldots w_{i_{k}}(e) w_{2}(e) w_{1}(e)\right) u_{12} \text {. }
$$

Очевидно, что слово $u_{11}\left(w_{i_{1}}(e) \ldots w_{i_{k}}(e) w_{1}(e) w_{2}(e)\right) u_{12}$ представимо в виде $w_{j_{1}}(e) \ldots$ $w_{j_{p}}(e)$ тогда и только тогда, когда в таком же виде представимо слово

$$
u_{11}\left(w_{i_{1}}(e) \ldots w_{i_{k}}(e) w_{2}(e) w_{1}(e)\right) u_{12} .
$$

Следовательно, искомым представлением будет либо $f_{1}+\left(f_{2}+u_{11} h_{1} u_{12}\right)$, либо

$$
\left(f_{1}+u_{11}\left(y w_{1}(e) w_{2}(e)+y w_{2}(e) w_{1}(e)\right) u_{12}\right)+\left(f_{2}+u_{11} f u_{12}\right)
$$

Случай многообразия $\mathfrak{X}_{k}^{1}$ рассматривается аналогично. Так как

$$
Q_{i}(e) A^{\xi_{1}}(e) B^{\xi_{2}}(e) C(e) D(e)+Q_{j}(e) A^{\eta_{1}}(e) B^{\eta_{2}}(e) C(e) D(e)
$$

- многочлен, состоящий из одночленов вида $w_{1}(e) \ldots w_{n}(e)$, ввиду свободы кольца $F \Re_{2}$ получаем, что в кольце $F \Re_{2}$ равенство (3) выполняется тогда и только тогда, когда в этом кольце справедливо равенство

$$
Q_{i}(e) A^{\xi_{1}}(e) B^{\xi_{2}}(e) C(e) D(e)+Q_{j}(e) A^{\eta_{1}}(e) B^{\eta_{2}}(e) C(e) D(e)=f_{1} .
$$

Индукцией по числу $d$ одночленов в многочлене $f_{1}$ покажем, что существует конечное вычисление на машине $\mathrm{M}$, переводящее конфигурацию $q_{i} \xi_{1} \xi_{2}$ в $q_{j} \eta_{1} \eta_{2}$, либо наоборот. Пусть $g$ - многочлен, полученный из многочлена (4) конечным числом перестановок вида

$$
\begin{array}{ll}
y A(e) B(e)=y B(e) A(e), & y A(e) D(e)=y D(e) A(e), \\
y B(e) C(e)=y C(e) B(e), & y C(e) D(e)=y D(e) C(e) .
\end{array}
$$

Пусть в кольце $F \Re_{2}$ вьполняется равенство $g=f_{1}$ и число одночленов в многочлене $f_{1}$ равно 0. Тогда утверждение очевидно. Допустим, что утверждение справедливо для некоторого числа $d$ одночленов. Покажем, что оно верно и для $d+1$. В самом деле, пусть в кольце $F \Re_{2}$ вьполняется равенство $g=f_{1}$ и число одночленов в многочлене $f_{1}$ не превосходит $d+1$. Так как $g=f_{1}$ - равенство в кольце $F \Re_{2}$, существует многочлен $v_{i 1} h_{j} v_{i 2}$ из суммы $f_{1}=\sum_{i, j} v_{i 1} h_{j} v_{i 2}$ такой, что $g_{1}$ - одночлен многочлена $v_{i 1} h_{j} v_{i 2}$, полученньй из

$$
Q_{i}(e) A^{\xi_{1}}(e) B^{\xi_{2}}(e) C(e) D(e)
$$


перестановками вида (5). Допустим, что $g_{1}$ - одночлен многочлена $v_{11} h_{1} v_{12}$. Пусть многочлен $h_{1}$ соответствует тождеству многообразия $\mathfrak{X}_{k}^{2}$. Тогда

$$
\begin{aligned}
v_{11} h_{1} v_{12}= & w_{i_{1}}(e) \ldots w_{i_{r}}(e) w_{1}(e) w_{2}(e) w_{j_{1}}(e) \ldots w_{j_{p}}(e) \\
& +w_{i_{1}}(e) \ldots w_{i_{r}}(e) w_{2}(e) w_{1}(e) w_{j_{1}}(e) \ldots w_{j_{p}}(e)
\end{aligned}
$$

причем

$$
\left\{w_{1}(e), w_{2}(e)\right\} \in\{\{A(e), B(e)\},\{A(e), D(e)\},\{B(e), C(e)\},\{C(e), D(e)\}\} .
$$

Следовательно, $v_{11} h_{1} v_{12}=H_{1}+H_{2}$, где $H_{1}=g_{1}, H_{2}$ - одночлен, полученньй из $H_{1}$ при помоши перестановок вида (5). Поэтому в кольце $F \Re_{2}$ вьполняется равенство

$$
g+H_{1}+H_{2}=\sum_{i>1, j} v_{i 1} h_{j} v_{i 2},
$$

где многочлен $g+H_{1}+H_{2}$ удовлетворяет тем же условиям, что и $g$, а суммав правой части (6) имеет меньшее число одночленов, чем $f_{1}$. Отсюда по предположению индукции получаем, что требуемое вычисление на машине М существует. Пусть теперь многочлен $h_{1}$ соответствует тождеству многообразия $\mathfrak{X}_{k}^{1}$. Тогда

$$
v_{11} h_{1} v_{12}=w_{i_{1}}(e) \ldots w_{i_{p 1}}(e) w_{l_{1}}(e) \ldots w_{l_{p 2}}(e)+w_{j_{1}}(e) \ldots w_{j_{p 3}}(e) w_{l_{1}}(e) \ldots w_{l_{p 2}}(e)
$$

где

$$
\begin{aligned}
\left\{w_{i_{1}}(e) \ldots w_{i_{p 1}}(e), w_{j_{1}}(e) \ldots w_{j_{p 3}}(e)\right\} \in\{ & \left\{Q_{i}(e) C(e) D(e), Q_{j}(e) A^{\varepsilon_{1}}(e) B^{\varepsilon_{2}}(e) C(e) D(e)\right\} \\
& \left\{Q_{i}(e) A(e) D(e), Q_{j}(e) A^{1+\varepsilon_{1}}(e) B^{\varepsilon_{2}}(e) D(e)\right\} \\
& \left\{Q_{i}(e) B(e) C(e), Q_{j}(e) A^{\varepsilon_{1}}(e) B^{1+\varepsilon_{2}}(e) C(e)\right\} \\
& \left.\left\{Q_{i}(e) A(e) B(e), Q_{j}(e) A^{1+\varepsilon_{1}}(e) B^{1+\varepsilon_{2}}(e)\right\}\right\} .
\end{aligned}
$$

Допустим, что

$$
\left\{w_{i_{1}}(e) \ldots w_{i_{p 1}}(e), w_{j_{1}}(e) \ldots w_{j_{p 3}}(e)\right\}=\left\{Q_{i}(e) A(e) D(e), Q_{j}(e) A^{1+\varepsilon_{1}}(e) B^{\varepsilon_{2}}(e) D(e)\right\}
$$

Остальные три случая рассматриваются аналогично. Так как имеет место (7), то

$$
\begin{gathered}
H_{1}=Q_{i}(e) A(e) D(e) w_{l_{1}}(e) \ldots w_{l_{p 2}}(e) \\
H_{2}=Q_{j}(e) A^{1+\varepsilon_{1}}(e) B^{\varepsilon_{2}}(e) D(e) w_{l_{1}}(e) \ldots w_{l_{p 2}}(e) .
\end{gathered}
$$

Отсюда вытекает, что $\xi_{1} \neq 0, \xi_{2}=0$ и $(6)$, где $g+H_{1}+H_{2}-$ многочлен, полученньй из многочлена

$$
Q_{j}(e) A^{\xi_{1}+\varepsilon_{1}}(e) B^{\xi_{2}+\varepsilon_{2}}(e) C(e) D(e)+Q_{l}(e) A^{\eta_{1}}(e) B^{\eta_{2}}(e) C(e) D(e)
$$

при помоши перестановок вида (5), и, кроме того, существует команда, при помощи которой машина М переводит конфигурацию $q_{i} \xi_{1} \xi_{2}$ в $q_{j}\left(\xi_{1}+\varepsilon_{1}\right)\left(\xi_{2}+\varepsilon_{2}\right)$, или наоборот. 
Так как число одночленов в сумме $\sum_{i>1, j} v_{i 1} h_{j} v_{i 2}$ меньше, чем в исходной, то по предположению индукции получаем, что существует конечное вычисление на машине М, переводящее конфигурацию $q_{i} \xi_{1} \xi_{2}$ в $q_{l} \eta_{1} \eta_{2}$, или наоборот.

Лемма 3 доказана.

Завершим доказательство теоремы. Зафиксируем некоторое число $k>40+m$. Пусть $r(x)=x+m+100$ и

$$
\mathfrak{A}_{1}=\mathfrak{X}_{k_{0}}, \quad \mathfrak{A}_{2}=\mathfrak{X}_{r\left(k_{0}\right)}, \quad \mathfrak{A}_{3}=\mathfrak{X}_{r\left(r\left(k_{0}\right)\right)}, \ldots
$$

Введем многообразия

$$
\begin{aligned}
& \mathfrak{B}_{1}=\operatorname{var}\left(z\left(\left(\ldots\left(\left(y x_{1}\right) x_{2}\right) \ldots\right) x_{r\left(k_{0}\right)}\right)=0\right), \\
& \mathfrak{B}_{2}=\operatorname{var}\left(z\left(\left(\ldots\left(\left(y x_{1}\right) x_{2}\right) \ldots\right) x_{r\left(r\left(k_{0}\right)\right)}\right)=0\right), \\
& \mathfrak{B}_{3}=\operatorname{var}\left(z\left(\left(\ldots\left(\left(y x_{1}\right) x_{2}\right) \ldots\right) x_{r\left(r\left(r\left(k_{0}\right)\right)\right)}\right)=0\right),
\end{aligned}
$$

Заметим, что многообразие $\mathfrak{X}_{\alpha}$ по определению удовлетворяет тождеству

$$
z\left(\left(\ldots\left(\left(y x_{1}\right) x_{2}\right) \ldots\right) x_{r(\alpha)}\right)=0 .
$$

Следовательно,

$$
\mathfrak{A}_{i}=\mathfrak{X}_{\alpha} \subset \operatorname{var}\left(z\left(\left(\ldots\left(\left(y x_{1}\right) x_{2}\right) \ldots\right) x_{r(\alpha)}\right)=0\right)=\mathfrak{B}_{i} .
$$

Так как $s\left(x_{1}, \ldots, x_{\alpha}\right)$ содержит подслово вида $Y\left(x_{1}, \ldots, x_{\alpha}\right)$, то $\mathfrak{B}_{i} \subset \mathfrak{A}_{i+1}$. Следовательно, многообразия $\mathfrak{A}_{i}$ и $\mathfrak{B}_{i}$ образуют цепочку:

$$
\mathfrak{A}_{1} \subset \mathfrak{B}_{1} \subset \mathfrak{A}_{2} \subset \mathfrak{B}_{2} \subset \cdots .
$$

Поэтому достаточно доказать, что эквациональная теория многообразия $\mathfrak{B}_{i}$ разрешима, а эквациональная теория многообразия $\mathfrak{A}_{i}$ неразрешима.

Очевидно, что тождество $H(\vec{x})=0$ выполняется в многообразии

$$
\operatorname{var}\left(z\left(\left(\ldots\left(\left(y x_{1}\right) x_{2}\right) \ldots\right) x_{\alpha}\right)=0\right)
$$

тогда и только тог да, когда каждьй одночлен многочлена $H(\vec{x})$ содержит подслово вида $Y\left(x_{1}, \ldots, x_{\alpha+2}\right)$. В кольце $F \Re$, свободном в многообразии всех колец, проблема равенства разрешима [7]. Следовательно, существует алгоритм, определяющий по произвольному слову, содержит ли оно подслово $Y\left(x_{1}, \ldots, x_{\alpha+2}\right)$. Отсюда вытекает, что эквациональная теория многообразия $\mathfrak{B}_{i}$ разрешима. Из лемм 2 и 3 вытекает, что в многообразии $\mathfrak{A}_{i}$ тождество

$$
Q_{1}(x) A^{2^{n}}(x) C(x) D(x)=Q_{0}(x) A(x) C(x) D(x)
$$

выполняется тогда и только тогда, когда машина М, начав работать в конфигурации $q_{1} 2^{n} 0$, через конечное число шагов переходит в конфигурацию $q_{0} 10$. По определению машины М это равносильно соотношению $n \in P$. Отсюда в силу нерекурсивности множества $P$ следует, что эквациональная теория многообразия $\mathfrak{A}_{i}$ неразрешима.

Теорема доказана. 
ДокАЗАТЕЛЬСТво СЛЕДСТВИЯ. Воспользуемся строением схемно-альтернативной иерархии язьков $\mathrm{SA}$, введенной и изученной в работе [6]. Поскольку $\forall \mathfrak{A}_{i}$ содержит лишь теорию $0 \mathfrak{A}_{i}$, которая пуста ввиду отсутствия в сигнатуре констант и, следовательно, разрешима, то $\forall \mathfrak{A}_{i}$ - критическая теория многообразия $\mathfrak{A}_{i}$. Для того чтобы показать, что $\forall \mathfrak{A}_{i}$ - единственная критическая теория, ввиду строения иерархии теорий SA $\mathfrak{A}_{i}$ достаточно установить, что теория $\exists \neg \vee \wedge \mathfrak{A}_{i}$ разрешима. Пусть $\varphi-$ произвольное предложение языка $\exists \neg \vee \wedge$. Тогда $\varphi$ равносильно предложению вида $\bigvee_{j=1}^{k} \varphi_{j}$, где $\varphi_{j}$ является $\exists \neg \vee \wedge$-предложением. Допустим, что для любого $j$ предложение $\varphi_{j}$ содержит отрицание. Стало быть, для любого $j$ предложение $\varphi_{j}$ ложно на одноэлементном кольце. Следовательно, $\bigvee_{j=1}^{k} \varphi_{j}$, а значит и $\varphi$, ложно на $\mathfrak{A}_{i}$. Допустим, что существует $j_{0}$ такое, что $\varphi_{j_{0}}-$ предложение языка $\exists \wedge$. Тогда $\varphi_{i}$ истинно в произвольном кольце на нулевом наборе. Поэтому $\mathfrak{A}_{i}=\bigvee_{j=1}^{k} \varphi_{j}$, а значит, $\mathfrak{A}_{i} \models \varphi$.

Следствие доказано.

Автор благодарит Ю.М. Важенина за руководство работой.

\section{СПИСОК ЦИТИРОВАННОЙ ЛИТЕРАТУРЫ}

[1] Мальцев А. И. Тождественные соотношения на многообразиях квазигрупп // Матем. сб. 1966. T. 69. №1. C. 3-12.

[2] Коуровская тетрадь. 10-е изд. Новосибирск, 1986.

[3] Мурский В. Л. Несколько примеров многообразий полугрупп // Матем. заметки. 1968. T. 3. №6. С. $663-670$.

[4] Клейман Ю. Г. О тождествах в группах // Тр. ММО. 1982. Т. 44. С. 62-108.

[5] Попов В. Ю. Эквациональные теории многообразий метабелевых и коммутативных колец // Алгебра и логика. 1995. Т. 34. № 3. С. 347-361.

[6] Важенин Ю.М. Алгоритмические проблемы и иерархии языков первого порядка // Алгебра и логика. 1987. Т. 26. № 4. С. 419-434.

[7] ЖКуков А.И. Приведенные системы определяющих соотношений в неассоциативных алгебрах // Матем. сб. 1950. Т. 27. №2. С. 267-280.

Уральский государственный университет, г. Екатеринбург

Поступило 03.08 .95

Исправленный вариант

16.12.97 